\title{
S. I: hybridization of neural computing with nature-inspired algorithms
}

\author{
Hari Mohan Pandey ${ }^{1} \cdot$ Nik Bessis $^{1} \cdot$ Neeraj Kumar $^{2} \cdot$ Ankit Chaudhary $^{3}$ \\ Published online: 26 March 2021 \\ (c) The Author(s), under exclusive licence to Springer-Verlag London Ltd., part of Springer Nature 2021
}

Hybrid intelligent methods have proven their efficiency to solve NP-hard optimization problems. Nature-inspired method plays a significant role across domains to solve multi-criteria decision-making problems. With the development of many recent technologies such as deep learning, big data, and cloud computing, nature-inspired methods have become a subject of broad and current interest. Many recent key breakthroughs in science and engineering especially in improving computational ability of the systems are highly related to nature-inspired computing research. Over decades, multi-criteria decision-making problems are throwing new challenges to the researchers and scientist working in the field of intelligent computing. Several natures inspired algorithms have been proposed to solve these problems. In addition, the researchers have proposed hybridization of nature-inspired algorithms by mixing it with neural networks which not only solve the multi-criteria decision-making problems successfully but also, they significantly improve the overall system performance. This is evidenced by the rapidly growing number of research publications and by the availability of excessive related software systems. Now, the researchers have understood the necessity to develop hybrid algorithms combining neural networks with nature-inspired algorithm and integrate decision making into multi-criteria optimization, and the need for cross-fertilization between hybrid neural computing with nature-inspired algorithms and the multiple criteria decision-making communities has become apparent.

The main aim of this special issue was to bring new and exciting issues and advancement to continue the integration and blending of ideas between hybrid neural computing

Hari Mohan Pandey

Pandeyh@edgehill.ac.uk

1 Edge Hill University, Ormskirk, UK

2 Thapar Institute of Engineering and Technology, Patiala, India

3 University of Missouri, St. Louis, USA with nature-inspired algorithms and multiple criteria decision-making, and to stimulate engagement with the user community. Authors are invited to submit full papers on recent advances in the development and application of hybrid neural computing with nature-inspired algorithms, new horizons for multiple criteria decision-making, which may be pathfinders for a step-change in multidisciplinary decision-making or showcase development in the multiple criteria decision-making communities that have potential for blending with hybrid neural computing with natureinspired algorithms theme.

The following articles in this special issue introduce a variety of $\mathrm{AI}, \mathrm{ML}$ and Hybrid methods for develop of intelligent systems. Most of the articles in this special issue address the problem of intelligent system development.

The article title "Comparative analysis of time series model and machine testing systems for crime forecasting" by Sudan Jha, Eunmok Yang, Alaa Omran Almagrabi, Ali Kashif Bashir and Gyanendra Prasad Joshi presents a strategy for a time series model and machine testing systems for crime estimation. Different techniques were applied to forecast the number of possible crimes in the next five years. Initially, existing machine learning methods were implemented for prediction of the number of crimes. Then, two approaches were proposed by modifying autoregressive integrated moving average model and modified artificial neural network model. This article also compares the applicability of a univariate time series model against that of a variate time series model for crime forecasting.

The article title "Nature-inspired algorithm-based secure data dissemination framework for smart city networks" by Kashif Naseer Qureshi, Awais Ahmad, Francesco Piccialli, Giampaolo Casolla and Gwanggil Jeon discussed three areas of the smart cities such as smart mobility, smart living and security provision using natureinspired algorithms. Three solutions were proposed, namely dragon clustering mobility in IoV, moth flame electric management for smart living and ant colony-based intrusion detection system for security provision. Simulation results demonstrated that the proposed algorithms 
were capable of secure data dissemination framework for smart city networks.

The article title "Deep Q-network-based multi-criteria decision-making framework for virtual simulation environment" by Hyeonjun Jang, Shujia Hao, Phuong Minh Chu, Pradip Kumar Sharma, Yunsick Sung and Kyungeun Cho has proposed a deep Q-network (DQN)-based multicriteria decision-making framework for virtual agents in real time to automatically select goals based on motivations in virtual simulation environments and to plan relevant behaviors to achieve those goals. Motivations had been classified considering five-level Maslow's hierarchy of needs, and the virtual agents train a double DQN by big social data, select optimal goals depending on motivations, and perform behaviors relying on a predefined hierarchical task networks (HTNs). Experimental result revealed that the proposed framework showed effective results over the state-of-the-art methods.

The article title "VNE strategy based on chaos hybrid flower pollination algorithm considering multi-criteria decision making" by Peiying Zhang, Fanglin Liu, Gagangeet Singh Aujla and Sahil Vashisht proposed a design strategy of hybrid flower pollination algorithm (FPA) for virtual network embedding. Here, merits of the genetic algorithm (GA) and FPA are combined for solving discrete optimization problems. This article utilizes a chaos optimization method as a replacement of sequence-guided crossover process to strengthen the global search capability and reduce the probability of producing invalid individuals. Numerical results revealed that the proposed algorithm showed good performance for link load balancing, revenue-cost ratio, $\mathrm{VN}$ requests acceptance ratio, mapping average quotation, average time delay, average packet loss rate, and the average running time of the algorithm.

The article title "A novel equilibrium optimization algorithm for multi-thresholding image segmentation problems" by Mohamed Abdel-Basset, Victor Chang and Reda Mohamed presented an equilibrium optimization algorithm to find the optimal threshold value for a grayscale image. The performance of the proposed algorithm was compared against the other popular optimization algorithms such as whale optimization algorithm, bat algorithm, sine-cosine algorithm, salp swarm algorithm, Harris hawk's algorithm, crow search algorithm, and particle swarm optimization. Though the proposed algorithm didn't outperform but showed good results on various quality measures.

The article title "A deep learning-based hybrid model for recommendation generation and ranking" by $\mathrm{N}$. Sivaramakrishnan, V. Subramaniyaswamy, Amelec Viloria, V. Vijayakumar and N. Senthilselvan proposed a hybrid Bayesian stacked auto-denoising encoder (HBSADE) model. It was implemented to recognize the latent interests of the user and analyses contextual reviews that are performed through the matrix factorization (MF) method. Here, authors consider a multilayer neural network to manipulate the nonlinearities between the user and item communication from data. Experimental results showed that the proposed system outperformed over the existing state-of-the-art methods.

The article title "Lung nodules detection using semantic segmentation and classification with optimal features" by Talha Meraj, Hafiz Tayyab Rauf, Saliha Zahoor, Arslan Hassan, M. IkramUllah Lali, Liaqat Ali, Syed Ahmad Chan Bukhari and Umar Shoaib implemented a framework to precisely detect lungs cancer to classify the benign and malignant nodules. The performance was tested using the subset of the publicly available dataset, i.e., the Lung Image Database Consortium image collection (LIDCIDRI). Empirical analysis showed that the proposed system outperformed other techniques and provides $99.23 \%$ accuracy using a logit boost classifier.

The article title "Synergetic fusion of energy optimization and waste heat reutilization using nature-inspired algorithms: a case study of Kraft recovery process" by Smitarani Pati, Drishti Yadav and Om Prakash Verma proposed a novel energy management strategy of multiplestage evaporator (MSE). Three nature-inspired algorithms, namely GA, differential evolution (DE) and particle swarm optimization (PSO), were implemented to determine the optimal process parameters. Experimental results showed that the proposed method outperformed other methods implemented in this article.

The article title "Investigation of multiple heterogeneous relationships using a q-rung orthopair fuzzy multicriteria decision algorithm" by Zaoli Yang, Harish Garg, Jinqiu Li, Gautam Srivastava and Zehong Cao presented a novel decision algorithm based on q-ROF set to deal with these using interactive operators and Maclaurin symmetric mean (MSM) operators. Proposed algorithm was analysed and validated considering the feasibility, sensitivity and superiority.

The article title "Neural image reconstruction using a heuristic validation mechanism" by Dawid Połap and Gautam Srivastava has implemented a heuristic validation mechanism for training different types of neural networks in the problem of image reconstruction. A mathematical model was described and supported by experimental results on different datasets with complex analysis of different heuristics to explain the computational ability of the proposed system.

The article title "Detection of abnormal brain in MRI via improved AlexNet and ELM optimized by chaotic bat algorithm" by Siyuan Lu, Shui-Hua Wang and Yu-Dong Zhang has proposed a novel abnormal brain detection method for magnetic resonance image. In this method, 
extreme learning machine was optimized by chaotic bat algorithm to obtain better classification performance. Experimental results revealed that the proposed algorithm outperformed over the state-of-the-art algorithms.

The article title "A hybrid approach for search and rescue using $3 D C N N$ and PSO" by Balmukund Mishra, Deepak Garg, Pratik Narang and Vipul Mishra proposed a novel approach to search and rescue using automated drone surveillance. Here, a complex scene classification problem was solved using the proposed 3DCNN model. Authors have implemented a PSO algorithm for hyper-parameter tuning for getting the best value of multiple parameters used for training the model. Simulation results demonstrated very impressive performance and achieved $99 \%$ validation accuracy.

Publisher's Note Springer Nature remains neutral with regard to jurisdictional claims in published maps and institutional affiliations. 